\title{
Pilot Study of a "Large-Eye," Surgically Induced Dry Eye Rabbit Model by Selective Removal of the Harderian, Lacrimal, and Meibomian Glands
}

\author{
Arthur S. Polans ${ }^{a, b}$ Jonathan F. McAnulty ${ }^{c}$ Paul O. Phelps ${ }^{d}$ \\ Soesiawati R. Darjatmoko ${ }^{a, b}$ Christopher K. Burris ${ }^{a, b}$ Daniel M. Albert ${ }^{b, e}$ \\ ${ }^{\mathrm{a} D e p a r t m e n t}$ of Ophthalmology and Visual Sciences, University of Wisconsin, ${ }^{\mathrm{b}}$ McPherson Eye Research Institute, \\ University of Wisconsin, and ' Department of Surgical Sciences, School of Veterinary Medicine, University of \\ Wisconsin, Madison, WI, d Department of Ophthalmology, Albany Medical Center, Albany, NY, and e Casey Eye \\ Institute, Oregon Health and Science University, Portland, OR, USA
}

\section{Keywords}

Dry eye $\cdot$ Harderian gland $\cdot$ Lacrimal gland $\cdot$ Meibomian gland

\begin{abstract}
Background/Aims: Establish a reliable rabbit dry eye (DE) model. Methods: An interventional cohort study surgically removing glands contributing to the tear film. Eight rabbits were studied after removal of left lacrimal, Harderian, or both glands. Additional rabbits had Meibomian glands in the left eye thermally obstructed. All were followed for 10 weeks with phenol red thread (PRT) and slit-lamp examination with $2 \%$ fluorescein. We assessed corneal sensitivity using a Cochet-Bonnet esthesiometer. Outcome measures were severity/duration of reduced PRT, punctate epithelial erosions (PEE), and histologic evidence of corneal pannus. Results: Fluorescein staining demonstrated signs of dryness including PEE in all of the interventional eyes. The subjective measurement of epithelial erosions correlated with decreased tear production. PRT measurements in the control eyes averaged $31.54 \mathrm{~mm}( \pm 1.83)$ and $22.71 \mathrm{~mm}( \pm 1.60)$ in the eight left eyes, without loss of corneal sensitivity. Conclusions: Surgical removal of either the Harderian or lacrimal gland results
\end{abstract}

\section{KARGER}

(C) 2017 S. Karger AG, Basel

E-Mail karger@karger.com

www.karger.com/oop in statistically significant decreases in tear volume and the development of severe DE. Removal of both glands results in the occurrence of a DE of comparable severity/duration to removal of either the lacrimal or Harderian gland alone. Meibomian gland obstruction contributes less to the DE model.

(c) 2017 S. Karger AG, Basel

\section{Introduction}

In humans, the tear film has 3 distinct layers: (1) the mucous layer, secreted by the conjunctival goblet cells, (2) the aqueous layer, secreted by the lacrimal glands, and (3) the lipid layer, secreted by the Meibomian glands [1]. The Harderian gland exists in tetrapods (including reptiles, amphibians, birds, and fur-bearing mammals) containing a nictitating membrane. The Harderian gland is named after the Swiss anatomist who discovered it in the red deer in 1694 [2-4]. It is particularly large and well developed in rabbits and rodents. In fact, the taxonomic order lagomorpha refers to rabbits, and the word "lagos" means "hare" in Greek, and "lagophthalmos" translates to an inability to close the eye. The rabbit may have developed the well-lubricated cornea, which requires few
Daniel M. Albert, MD, MS

Oregon Health and Science University, Casey Eye Institute

3375 SW Terwilliger Blvd

Portland, OR 97239-4197 (USA)

E-Mail albedan@ohsu.edu 
Fig. 1. Representative corneal photographs of treated and control eyes. a Control. b Harderian gland removed. c Lacrimal gland removed. d Meibomian gland removed. e Both lacrimal and Harderian glands removed.

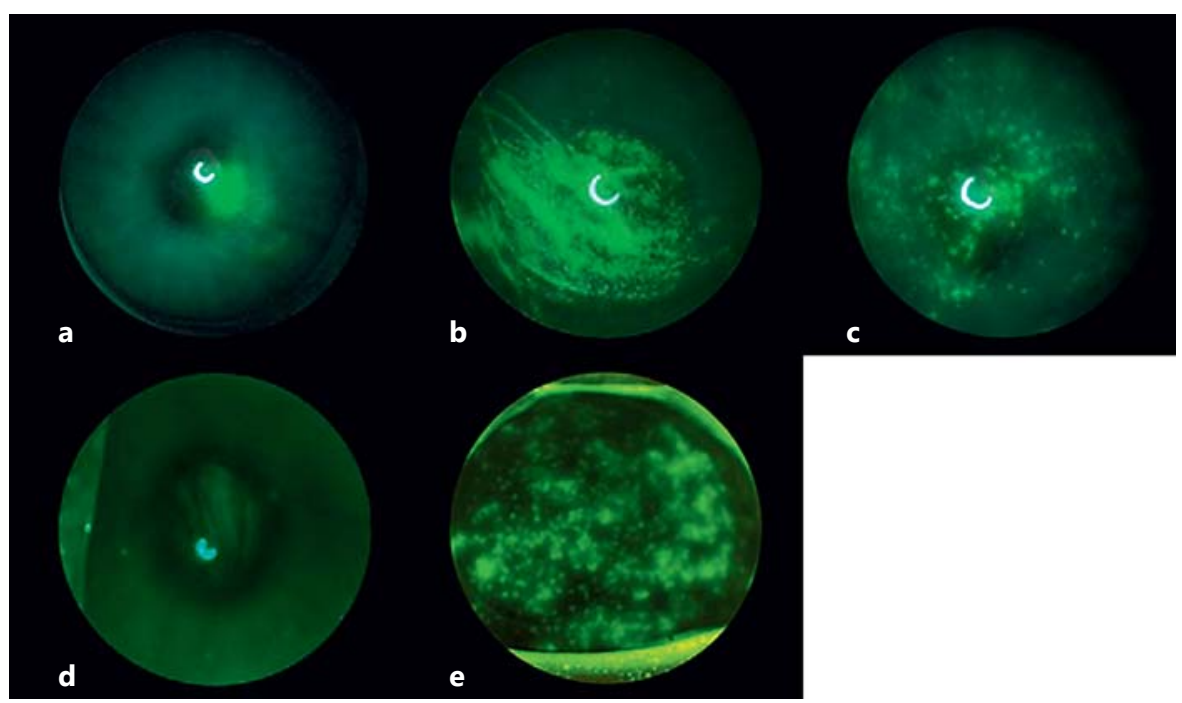

blinks to maintain the ocular surface, as an evolutionary adaptation because the lack of a blink may allow the rabbit to avoid detection by predators. While there are debates regarding its function in various species, it is known that the Harderian gland is involved in the production of unique lipids in rabbits [5]. One study found that 1-alkyl-2,3-diacylglycerol is a unique product of the Harderian gland in rats [6].

Several groups have attempted to make an effective and predictive animal dry eye model [7-11]. The fact that no standard model has emerged is evidence of their limitations. One major problem with previous animal models is that they demonstrate more mild forms of dry eye which can be made to resolve with the vehicle used to deliver prescription eye drops or OTC palliatives. Additionally, recent research has shown that compensatory mechanisms may increase the aqueous production in patients with long-standing Meibomian gland dysfunction [12], confounding strategies to specify the tear component responsible for ocular surface dryness. Although mouse models have been widely employed, the rabbit model has the advantage of a larger cornea, which allows photography and slit-lamp examination as well as optical coherence tomography like that done in the clinical setting. Most previous rabbit models have been pharmacologically or immunologically induced. Such models provide reproducible changes in tear volume but no dynamic range of fluorescein staining, thus limiting distinctions upon drug treatment. Previous efforts to create a surgically induced dry eye model in rabbits involved more conservative surgical approaches [7], presumably be- cause more complete resection of the lacrimal glands may result in puncturing the large orbital venous sinus and exsanguination of the animal.

\section{Methods}

Six male New Zealand albino rabbits had their lacrimal, Harderian, or both glands surgically removed from their left orbits via an anterior orbitotomy approach (one of the authors, J.F.M., developed a surgical technique which avoided exsanguination while addressing the orbital venous sinus). The Harderian glands were identified by their large size, medial location in the orbit (posterior to nictitating membrane), and unique pale white and pink lobes. The lacrimal glands were located in the anterior orbit and pinkish-yellow in color. Two male New Zealand albino rabbits had the Meibomian glands of their left upper and lower eyelids cauterized using a Prolectro epilator. The right eye was utilized as a control. The animals were followed by standard methods of fluorescein staining, phenol red thread (PRT), and histological analysis. A $\chi^{2}$ test was performed on the PRT measurements from the control and intervention eyes to determine the statistical significance of the difference in recorded values. Corneal sensitivity was determined using a Cochet-Bonnet esthesiometer, adjusting the length of the nylon probe in $5-\mathrm{mm}$ increments. The control right eyes (OD) were compared to the interventional left eyes (OS) to evaluate the status of corneal wetting and the clinical and histological evidence of corneal changes. After 10 weeks, the animals were sacrificed, and the corneas were fixed with formalin and processed using standard methods for histologic evaluation. Photomicrographs of the rabbit corneas were taken with an Aperio from Leica Biosystems. An area with changes corresponding to human pannus was present at the limbus. The corneal pannus of each cornea was measured, in a masked fashion, from the end of Descemet's membrane to the most anterior corneal blood vessel. The measurements were taken at 12 o'clock and 6 o'clock for each eye by marking the 12 o'clock position prior to enucleation. 


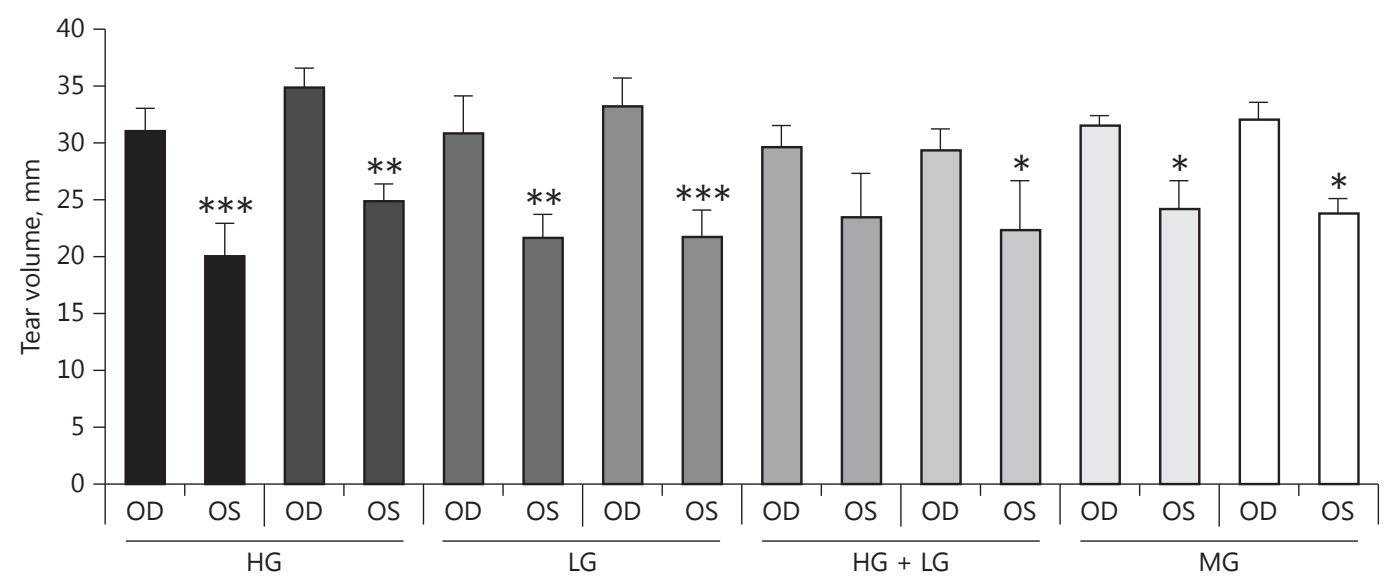

Fig. 2. Measurements of tear volume in control and surgically treated eyes. The $y$ axis indicates the phenol red thread measurement in $\mathrm{mm}$ which has been averaged over 10 weeks (mean $\pm \mathrm{SD}$ ). ${ }^{*} p \leq 0.05,{ }^{* *} p \leq 0.01,{ }^{* * *} p \leq 0.001$. HG, Harderian gland removed; LG, lacrimal gland removed; MG, Meibomian gland removed.

Fig. 3. A measurement was taken from the end of Descemet's membrane to the most anterior corneal blood vessel in each eye. These measurements were thought to be more reliable than measuring from the corneal limbus because of the variable amount of conjunctival folds, as seen below.

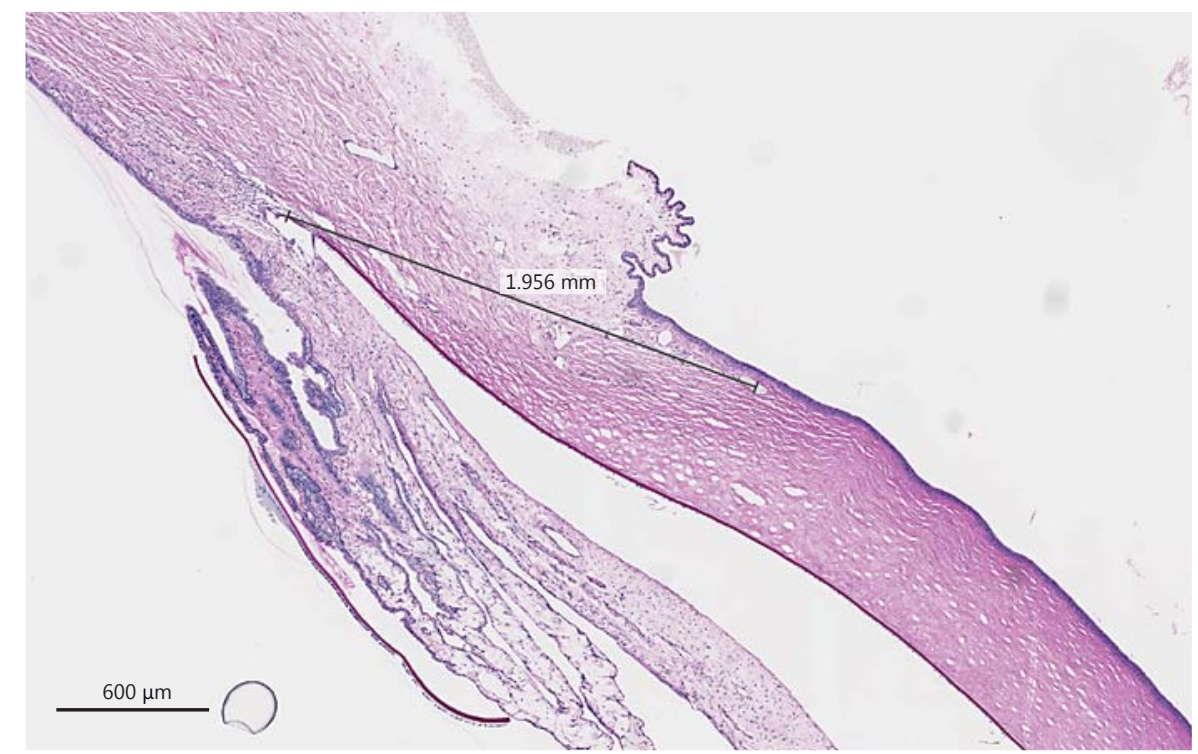

\section{Results}

Fluorescein staining demonstrated signs of corneal dryness in all of the interventional eyes (Fig. 1). During the first 4-6 weeks, the staining tended to be variable. Thereafter, the corneal staining became more consistent and severe in the interventional eyes compared with the nonsurgical eyes (Fig. 1a). The cornea of rabbits with the Harderian gland removed demonstrated denser, geographic-appearing punctate epithelial erosions in the central cornea (Fig. 1b). The left corneas of the rabbits with their lacrimal glands removed tended to have more diffuse punctate epithelial erosions (Fig. 1c). The rabbits with Meibomian gland cauterization demonstrated diffuse punctate epithelial erosions, similar to, but fewer than, the rabbits with lacrimal gland removal (Fig. 1d). The rabbits with both glands removed tended to have a combination of both patterns of staining, with focal areas of dense epithelial erosions with diffuse punctate epithelial erosions (Fig. 1e).

To ascertain whether damage to the corneal epithelium following surgery resulted from impaired corneal in- 
nervation, similar to what occurs in such diseases as neurotrophic keratopathy, a Cochet-Bonnet esthesiometer was used to evaluate corneal sensitivity as a measure of the health of the ophthalmic branch of the fifth cranial nerve, which carries sensory fibers for the cornea. Measurements were made at the center of each cornea from the surgical eye and the companion nonoperated eye in each of three rabbits. Corneal sensitivity upon reaching one-quarter lid closure was $50.0 \pm 5.0 \mathrm{~mm}$ (mean $\pm \mathrm{SD}$ ) in the operated eyes and $33.3 \pm 10.4 \mathrm{~mm}$ in the nonoperated eyes. At full closure, the values for the operated and nonoperated eyes were $33.3 \pm 2.9$ and $20.0 \pm 1.0 \mathrm{~mm}$, respectively. Measurements at both levels of lid closure therefore demonstrated that operated eyes were equal to or more sensitive than nonoperated eyes, confirming that nerve damage was not the cause of changes to the integrity of the corneal epithelium following surgery.

Tear production, as measured by PRT, demonstrated a reproducible decrease in the left eye of all rabbits in this study. In the control eye of the 8 rabbits, the PRT measurements averaged $31.54 \mathrm{~mm}( \pm 1.83)$ over the 10 weeks of follow-up measurements. In the 8 operated left eyes, the PRT measurements averaged $22.71 \mathrm{~mm}( \pm 1.60)$. The difference between the PRT measurements in the intervention eyes and the control eyes were found to be statistically significant $(p \leq 0.05)$ in all but one rabbit. The average tear production over the course of 10 weeks and $p$ values can be seen in Figure 2. While changes in tear volume were comparable between those eyes in which the Harderian or lacrimal glands had been removed, Meibomian gland disruption showed less effect (Fig. 2).

The histopathologic changes in the rabbit corneas were minimal. The epithelium appeared to be generally intact (Fig. 3). However, there was a trend towards an increased corneal pannus in the interventional left corneas. The mean corneal pannus in the untreated right eye of rabbits with their Harderian glands removed was 1.88 $\mathrm{mm}( \pm 0.15 \mathrm{SD})$, while in the interventional left eye, it was $2.05 \mathrm{~mm}( \pm 0.12 \mathrm{SD})$. The mean corneal pannus in the untreated right eye of rabbits with their lacrimal glands removed was $1.79 \mathrm{~mm}( \pm 0.16 \mathrm{SD})$, while in the interventional left eye, it was $2.06 \mathrm{~mm}( \pm 0.07 \mathrm{SD})$. The mean corneal pannus in the untreated right eye of rabbits with both their Harderian and lacrimal glands removed was 1.68 $\mathrm{mm}( \pm 0.31 \mathrm{SD})$, while in the interventional left eye, it was $1.61 \mathrm{~mm}( \pm 0.47 \mathrm{SD})$. The mean corneal pannus in the untreated right eye of rabbits with both their Harderian and lacrimal glands removed and their Meibomian glands cauterized was $1.88 \mathrm{~mm}( \pm 0.05 \mathrm{SD})$, while in the treated left eye, it was $1.56( \pm 0.13 \mathrm{SD})$.

Dry Eye Rabbit Model

\section{Conclusions}

The removal of the lacrimal, Harderian, or both glands results in a severe dry eye model. Cauterization of the Meibomian glands also demonstrates changes consistent with chronic dry eye. Previous dry eye models have failed to reproduce the complex disease state that is seen in human dry eye. However, the large cornea of rabbits makes it ideal for clinical monitoring, as seen in our study. Importantly, an animal model of moderate to severe dry eye is invaluable in testing novel compounds that may require delivery vehicles, such as emulsified castor oil or glycerin, which may cure mild dry eye of other models. These studies confirm that the Harderian gland secretions are necessary for the prevention of dry eye in rabbits, and surgical removal of the gland results in an animal model with moderate to severe dry eye, which should be useful in testing lipid and other hydrophobic compounds intended to treat dry eye.

\section{Acknowledgements}

This study was supported in part by the Core Grant for Vision Research from the NIH to the University of Wisconsin-Madison (P30 EY016665) and an unrestricted grant to the Department of Ophthalmology and Visual Sciences from Research to Prevent Blindness, Inc. The authors thank Laura Cruz for her assistance in the preparation of this article.

\section{Statement of Ethics}

This study adhered to the ARVO Statement for the Use of Animals in Ophthalmic and Vision Research and NIH guidelines for the use of animals in research and was approved by the University of Wisconsin, School of Medicine and Public Health Animal Care and Use Committee.

\section{Disclosure Statement}

The authors declare that there are no conflicts of interest. 


\section{References}

1 Moshirfar M, Pierson K, Hanamaikai K, Santiago-Caban L, Muthappan V, Passi SF: Artificial tears potpourri: a literature review. Clin Ophthalmol 2014;8:1419-1433.

2 Albert DM, Frayer WC, Black HE, Massicotte SJ, Sang DN, Soque J: The harderian gland: its tumors and its relevance to humans. Trans Am Ophthalmol Soc 1986;84:321-341.

3 Hillenius WJ, Phillips DA, Rehorek SJ: “A new lachrymal gland with an excretory duct in red and fallow deer" by Johann Jacob Harder (1694): English translation and historical perspective. Ann Anat 2007;189:423-433.

4 Payne AP: The harderian gland: a tercentennial review. J Anat 1994;185:1-49.
5 Bayraktaroglu AG, Ergun E: Histomorphology of the Harderian gland in the Angora rabbit. Anat Histol Embryol 2010;39:494-502.

6 Miyazaki M, Kim HJ, Weng CM, Ntambi JM: Oleoyl-CoA is the major de novo product of stearoyl-CoA desaturase 1 gene isoform and substrate for the biosynthesis of the Harderian gland 1-alkyl-2,3-diacylglycerol. J Biol Chem 2001;276:39455-39461.

7 Gilbard JP, Rossi SR, Gray KL: A new rabbit model for keratoconjuntivitis sicca. Invest Ophthalmol Vis Sci 1987;28:225-228.

8 Gilbard JP, Rossi SR, Heyda KG: Tear film and ocular surface changes after closure of the meibomian gland orifices in the rabbit. Ophthalmology 1989;96:1180-1186.

9 Barabino S, Dana MR: Animal models of dry eye: a critical assessment of opportunities and limitations. Invest Ophthalmol Vis Sci 2004; 45:1641-1646.
10 Chen Z, Liang QF, Yu GY: Establishment of a rabbit model for keratoconjunctivitis sicca. Cornea 2011;30:1024-1029.

11 Li N, Deng X, Gao Y, Zhang S, He M, Zhao D: Establishment of the mild, moderate, and severe dry eye models using three methods in rabbits. BMC Ophthalmology 2013;12:50.

12 Arita R, Morishige N, Koh S, Shirakawa R, Kawashima M, Sakimoto T, Suzuki T, Tsubota K: Increased tear fluid production as a compensatory response to meibomian gland loss: a multicenter cross-sectional study. Ophthalmology 2015;122:925-933. 